\title{
Parts of the Whole: Moderating Competition in the World of Ideas
}

\section{Dorothy Wallace}

Dartmouth College, dorothy.wallace@dartmouth.edu

Follow this and additional works at: https://digitalcommons.usf.edu/numeracy

Part of the Higher Education Commons, and the Science and Mathematics Education Commons

\section{Recommended Citation}

Wallace, Dorothy. "Parts of the Whole: Moderating Competition in the World of Ideas." Numeracy 8, Iss. 1 (2015): Article 14. DOI: http://dx.doi.org/10.5038/1936-4660.8.1.14 


\title{
Parts of the Whole: Moderating Competition in the World of Ideas
}

\author{
Abstract \\ In both biological and mathematical dynamical systems, competition may be moderated by the presence \\ of a top predator of both competing species. Applying this principle in memetics sheds light on why some \\ strategies are more likely than others to promote quantitative literacy in various kinds of institutions. \\ Keywords \\ numeracy, quantitative literacy, postsecondary education, memetics, dynamical system, competition \\ Creative Commons License \\ (c) (i) (8) \\ This work is licensed under a Creative Commons Attribution-Noncommercial 4.0 License

\section{Cover Page Footnote} \\ Dorothy Wallace is a professor of mathematics at Dartmouth. She was 2000 New Hampshire CASE \\ Professor of the Year, and the lead PI of the seminal NSF project, Mathematics Across the Curriculum. \\ She recently finished a text in mathematical biology for first-year students, "Situated Complexity." She was \\ a charter board member of the National Numeracy Network and is now co-editor of this journal.
}




\section{Parts Of The Whole A Column by $\mathrm{D}$. Wallace}

The problem of how best to improve the numeracy of a society is a thorny one, embracing the learning process of a single student but rising in scale to include the management and alteration of an entire system of education. With the issue of quantitative literacy always in mind, this column considers various aspects of the systemic workings of education, the forces acting on classrooms, teachers and students, and mechanisms of both stasis and change.

\section{Moderating Competition in the World of Ideas}

A mathematical understanding of stability makes in clear that, in many cases, a diversity of ideas is likely to be stable in the world of memes. But because unsuccessful ideas are often forgotten, we must remember that the prognosis for survival may be poor for any particular meme. The notion that education should result in quantitative literacy is a new idea introduced into the academy, itself a whole world of ideas. At the outset one would suspect numeracy doesn't have much of a chance, unless special care is taken.

For practical purposes one would wish to know from the ecologist what mechanisms make survival possible on a species by species basis. Ecology and mathematics again offer specific scenarios under which competition between a pair of species is a stable situation. One scenario uses a third species to moderate competition by functioning as a top predator. Here we look at this mechanism and how it functions mathematically and in a biological system. This insight suggests strategies for promoting quantitative literacy in a variety of institutions.

\section{The Top Predator in a Dynamic Model}

In this scenario we begin with the classic equations for two species in competition for a single resource. Let us assume that, barring other influences, mathematics will dictate that one species will outcompete the other; the loser becoming extinct after a period of time.

To these equations we add some more that represent a top predator, which eats both competing species according to their relative abundance, perhaps with some preference for the stronger competitor. It is easy to see heuristically how the top predator functions to keep both species alive. When the less-competitive species becomes scarce, the top predator eats only the more-successful species, giving the distressed population time to recover. Heuristics are borne out in the math. The top predator, by always preferring the lazy route, avoids decimating 
either species. Remove the top predator and one of the two competitors will become extinct. At least, that is how the mathematics works.

\section{The Top Predator in Nature}

This scenario is borne out in nature. E. Wilson's The Diversity of Life contains a description of the ecosystem of the kelp beds off the coast of California. This large and complex ecosystem contains two stable subsystems. One has algae, fish, shellfish, and almost all of the creatures in the waters, excepting sea urchins. The other stable system contains only algae and sea urchins. The sea urchins are voracious consumers of algae, and nothing can eat them except sea otters. If we think of the graph-theoretic representation of the whole system, the sea otter is the top predator and the rest of the graph splits into two nearly independent parts. One contains the sea urchin and the other contains nearly everything else. These two parts, one huge and one small, are reunited at the bottom of the food chain at the node of the graph corresponding to algae. So the sea urchins and most of the rest of the ecosystem function as a pair of competing species. If the top predator, the sea otter, were removed, we might expect one "species" to go extinct, either the sea urchin or the interconnected collection of diverse interrelated organisms in competition with it.

Early in this century humans ran this experiment. Fur traders trapped sea otters. Fishermen destroyed what the trappers didn't get, perceiving the otters to be in competition with them. In short order, fishing became poor. The waters became completely infested with sea urchins, to the exclusion of all else. Without a top predator, the sea urchin outcompeted the entire rest of the ecosystem. In accord with the prognosis of the dynamical systems models of the relative stability of diverse versus simple ecosystems, the simpler system prevailed.

\section{The Top Predator in the World of Memes}

Because of the fluidity with which ideas merge and change, it is difficult to come up with a memetic parallel to this situation. Few ideas function as "top predators," and it is difficult even to formulate a definition of what that might mean in the context of interacting ideas. A useful interpretation of "top predator" might be an idea or set of beliefs so forceful that it supercedes all (or most) other considerations. The example given next shows both how such a mechanism would work and also some of its limitations.

Astrology was once a respectable part of scientific pursuit. Indeed, astrological considerations fostered the growth of both astronomy and mathematics for most of the history of these two subjects. Scientists however are remarkable in their denial of their own history, and now one often hears astrology

\footnotetext{
${ }^{1}$ Wilson, Edward O. The diversity of life. WW Norton \& Company, 1999.
} 
called a "pseudoscience" by today's scientists. The scientific mindset is a celebrated attitude toward knowledge on which most modern advances rest. Yet astrology has no shortage of practitioners, advocates, and even true believers in this most modern moment in history (to date).

Many scientists denounce astrology as a pseudoscience largely because there is almost no evidence that it works. Go on, they urge, check your horoscope every day and see what percent of the time it says anything the least bit accurate about you. Collect data, they say with confidence, because many others have done so and you too will see that there is no good correlation between your life and the predictions of your horoscope. A theory for which there is no supporting data must be discarded. This is the scientific meme at work.

Lovers of astrology remain unconvinced by this argument. Just because horoscopes don't work for you doesn't mean they won't work for me. Just because they did not work yesterday doesn't mean they won't work tomorrow. People have respected astrology for far longer than they have respected modern science, and not all of those people were idiots. When cornered, a horoscope aficionado may even utter the sentence most likely to cause a scientist to cringe: "It makes as much sense as most religions." They rarely utter the phrase: "Absence of evidence is not evidence of absence," a statement sometimes heard by statisticians.

Here we have powerful ideas opposed in competition for the belief system of an individual. Recent advances oppose a long tradition. Belief in data and its reliability opposes belief in ancient wisdom. Both beliefs exist in most cultures; both are useful. The mechanism preventing one of these belief systems from stamping out the other must be a set of ideas that dominates both the modern reliance on data and the reverence for ancient wisdom. This mechanism is so old, so venerated, so embedded in us that we usually take in for granted.

It is logic. Proof by deductive logic is still the kind of argument that holds fast. While scientists may correctly claim that there is no proof that astrology works, lovers of astrology can equally well claim that there is no proof that astrology cannot possibly work. Both belief systems are held in check by a notion of deductive proof that moderates both the respect for ancient knowledge and the modern reliance on data. Deductive proof, or in this case lack thereof, functions as a "top predator" in this situation.

\section{Finding the Top Predator}

If we are to find other examples of ideas, so solidly embedded in our beliefs, that they are in a position to moderate the competition between less-entrenched ideas, then we must look very deep. Such beliefs must be nearly unquestionable by an entire culture and are therefore often taken for granted. They might not even be spoken aloud, even in the culture of a university where it seems that everything is discussed constantly. 
What ideas then might function as a top predator in discussions of quantitative reasoning at a college or university? It depends on the nature of the institution, whose overriding concerns should be expressed in its mission statement.

\section{Public Colleges and Universities}

Let's look first at a public institution driven by accountability to state governments wishing to improve the local economy. The big idea here is that students should leave equipped for jobs in local industries, perhaps able to start businesses of their own, or enter legal or medical professions. The general life competency of a person should be enhanced by his or her college education. As an instructor of a particular course in a particular department, it is easy to lose sight of this goal. But quantitative reasoning can be packaged to address such a goal directly.

One possible path is to recognize that most businesses and professions routinely rely on the use of spreadsheets to track expenses, make projections, and create reports for investors or administrators. Yet one rarely sees courses in the use of spreadsheets, and certainly not as a requirement. Such a course, done through project-based learning, could be a magnificent vehicle for boosting quantitative reasoning. Students would have to understand basic algebra enough to program a spreadsheet. They would grapple with quantitative aspects of a well-designed project, such as unit conversion or estimation. Finally they would improve their ability to understand charts and graphs by creating ones that support their conclusions or solution to a problem. Even if only the business majors at a large university took such a course, it would make a formidable contribution to the quantitative skills of a large population. The idea behind the course is clearly aligned with the goals of the institution, without directly threatening any potential competing use of resources.

Another possible gateway to quantitative literacy is the ubiquitous personal finance course. This course is also often taught in business departments, and many institutions are starting to require some kind of financial training of all students. These institutions recognize that, although a college education is beneficial in the workplace, its value may be offset by the financial troubles students suffer from not understanding how their student loans and credit cards work. Careless financial behavior in college can dog a person for many years after graduation, interfering with the ability to function fully in the workplace. At present, many personal finance courses do not stress the more-quantitative aspects of money. This is an opportunity for those promoting quantitative reasoning to step in and offer to build a far better kind of personal finance course in which people learn to use their math to make important decisions in life. A beefed up personal finance course that satisfies a quantitative requirement would be a natural way to appeal both to students and to the larger needs of their institution. 


\section{The Liberal Arts College}

Now let us consider a private liberal arts institution that envisions creating a discerning citizen. The mission of this institution may not align directly with courses aimed at the same sort of practical, almost vocational, knowledge that would appeal in other places. Yet quantitative reasoning is well positioned to serve the goal of creating a responsible citizenry. Political arguments and governmental decisions at all levels are informed and misinformed by data-driven arguments. Quantitative literacy is the key to understanding when a numbers argument is close to correct, and when it is an outright fallacy. Issues of fairness must be argued with numbers. Solutions to every civic issue have associated costs, and it requires quantitative reasoning to estimate them.

The idea that every college-educated person should be able to read the newspaper critically is a deeply quantitative proposition. It also aligns well with the goals of a liberal arts institution. Advice on how to structure such a course is available on the National Numeracy Network web site, based on Madison's ongoing course at the University of Arkansas. All of the topics in that course are drawn from recent newspaper articles. Originally framed as a course for journalists, it could easily be a general quantitative education offering. Those familiar with the Chance course and materials will recognize the format. The Chance course used newspaper articles as a source of interesting questions in probability. It was quite successful and is still offered regularly in many institutions. The instructor needs to think on his or her feet, but it is also possible to prepare well in advance if one is willing to use newspaper topics that are a few months old.

Liberal arts colleges often have a service component built into their mission statements. Service education is an enormous opportunity for students to master a part of quantitative literacy. A deep analysis of what it takes to solve a civic or small-business problem always involves costs, time estimates, labor estimates, and often estimates of materials as well. If the intervention creates an ongoing effort, then figuring out how to make that effort sustainable in the long run is in part a quantitative problem. A course designed entirely around civic projects should include such questions as a natural part of any project, and it would serve the quantitative education of students very well.

\section{Schools of Science and Engineering}

What about an institution priding itself on science and engineering? The students of this institution are being prepared in a very direct manner for technical careers. The concerns of the large public university and the liberal arts college are not the concerns of this institution. Here however, it is easy to argue that quantitative reasoning, suitably interpreted, builds better scientists.

The mathematics that we use to arrive at many scientific conclusions is of primary importance. But so are the back-of-the envelope estimates that we use to 
double check that we are getting reasonable answers. A novice can make the simple mistake of moving a decimal point, and will not notice that the answer is an order of magnitude off. An expert will notice, because of the kind of simple estimates and comparisons that one would do if more-sophisticated mathematics were not available. The difference between novice and expert is a very useful kind of quantitative literacy that would serve young scientists well. Quantitative reasoning of this sort could and should be built into every course.

Students majoring in less mathematically intensive subjects, such as biology or earth science, may miss an important path to understanding. The act of modeling a biological system with ordinary differential equations, for example, requires understanding that system at a deep level. The relationships among organisms are embodied in the terms of such an equation, and require thoughtfulness at every step. The computer is a friend here, allowing students with minimal calculus background to simulate biological systems and feel the power of mathematics. Once the basic equations are in place, figuring out what the parameters ought to be is an enormous exercise in quantitative reasoning. Unit conversion is paramount. Deciding on appropriate units is difficult (Number of individuals in the pond? Number per cubic meter? Biomass per cubic meter? Percent change of biomass per cubic centimeter per day? Wet biomass or dry biomass?). Most difficult of all is estimating these numbers based on either experiments or field data. All of a sudden, the amount of semi-digested insect matter in the gut of a frog has a new, and very technical, quantitative meaning. After the model is constructed and it is producing answers, one should be able to argue that the answers are indeed reasonable in comparison with reality. Only parts of reality are usually measured, and often only in comparative terms. It can require not only quantitative reasoning, but also some creativity, to figure out if these measurements align with a model. Students who can do these things will be better scientists, and this fact may be argued in complete alignment with the goals of the institution.

\section{How NOT to Argue for Quantitative Literacy}

All of the examples above are presented in the context of a particular kind of institution. None of these arguments degrade or compete with any other subject. All of them appeal to the overriding mission of that institution, which one hopes will serve as the "top predator" in mediating any perceived or actual competition that may arise with other demands. New ideas may need some protection to thrive, and quantitative literacy is no exception. If we frame numeracy so that it is central to the mission of our institution, we have far better chances of success.

One argument that is sometimes given in favor of courses in quantitative reasoning highlights the principles in this column. Sometimes people make this claim: "Quantitative literacy is likely to be far more useful to students than the usual college algebra class." This line of reasoning, whether true or false, is 
actually detrimental to the acceptance of quantitative reasoning in an institution. Let us see why.

First of all, the statement implicitly places quantitative literacy in the domain of mathematics. The argument becomes, implicitly, about what should be taught as a mathematics requirement, and probably by the mathematics department. Put this way, the argument is no longer in the context of the larger goals of the college or university, but rather about distributional requirements relating to math. What functions as the "top predator" in this universe of ideas? Some science departments will have strong mathematics requirements for their students, none of which currently resemble a quantitative reasoning course. They know exactly why algebra is needed. The mathematics department itself will have the opinion that it is there to teach mathematics, and not some fuzzy alternative. In short, the big ideas local to the STEM disciplines will not make it easy for a quantitative reasoning course to thrive as an alternative to college algebra.

Second, by being placed in opposition to algebra, quantitative reasoning is implicitly placed in opposition to a strong STEM track for future scientists. Students from disadvantaged backgrounds who come somewhat later to math and science include underrepresented groups that, at a slightly higher level, are actively recruited by the STEM disciplines. Tracking these people away from a STEM path early in their college careers potentially does a deep disservice to the STEM disciplines and the nation as a whole. Quantitative literacy was never intended to do that, and an argument that suggests a tracking of this sort cannot succeed in the long run, because the "top predator" in this context is the admirable idea that science is open to all.

Third and most obvious, the argument as stated above is a direct competitive challenge to an established meme (algebra). Even if the argument that "quantitative literacy is more useful than algebra" were correct (which is certainly not being argued here), it is a poor strategy because it takes a competitive stance in the first place.

\section{A Good General Strategy}

The strategy for embedding quantitative literacy as a desired outcome for all college students, with courses supporting that end, should be informed by our best understanding for how new ideas come to be deeply accepted by a culture. This understanding, in turn, comes from insights drawn from biology and supported by mathematics. One of these, the notion of the "top predator" that mediates competition for resources, has direct analogues in the world of ideas. These are the big ideas that drive the very nature and mission of an institution.

In summary, the discussion presented here suggests a strategy that appeals to the core mission of an institution, that rises above the concerns of a particular department or division, and that does not place itself as an obvious competitor to a particular established course of study. The ease with which arguments of this sort 
Numeracy, Vol. 8 [2015], Iss. 1, Art. 14

may be made highlights the relevance and importance of quantitative literacy as part of the curriculum in any college or university. 